\title{
PRIMER REGISTRO DE GENLISEA (LENTIBULARIACEAE) PARA MÉXICO
}

\author{
Martha Olvera García \\ Y \\ Esteban Martínez S. \\ Herbario Nacional de México \\ Instituto de Biología, UNAM \\ Apartado postal 70-367 \\ 04510, México, D.F.
}

\section{RESUMEN}

Se registra por primera vez para México la presencia de Genlisea filiformis A. St.-Hil. Se mencionan sus características distintivas, y se proporciona información acerca de su hábitat y distribución geográfica.

Palabras clave: Chiapas, Genlisea, Lentibulariaceae, México, planta insectívora.

\begin{abstract}
Genlisea filiformis A.St.-Hil. is reported for the first time from Mexico. Its distinctive characters, as well as information on its habitat and geographic distribution are presented.

Key words: Chiapas, Genlisea, Lentibulariaceae, Mexico, insectivorous plant.

Durante la revisión de material herborizado de Utricularia, colectado en el estado de Chiapas por el segundo autor para la Flora Mesoamericana, se encontraron mezclados algunos ejemplares del género Genlisea que no se había registrado para el territorio mexicano y que por sus características morfológicas corresponden a Genlisea filiformis.

Genlisea es un género de regiones tropicales, cuyo centro de diversificación es Sudamérica. Se distingue fácilmente de los otros miembros de la familia Lentibulariaceae por el cáliz 5-partido y las hojas insectívoras tubulares que terminan en dos brazos helicoidales (Cuadro 1).
\end{abstract}

Genlisea filiformis A. St.-Hil., Voy. Distr. Diam. 2: 430. 1833. Genlisea luteo-viridis Wright, Anales de la Academia de Ciencias Médicas 6: 319. 1869. Genlisea anfractuosa Tutin, J. Bot. 72: 310. 1934.

Las plantas pertenecientes a esta especie son hierbas terrestres, delicadas, de 5 a $10 \mathrm{~cm}$ de largo; se distinguen por la presencia de pelos glandular-capitados en la 
inflorescencia y el espolón obtuso más largo que el labio inferior. Por la corola amarilla y los frutos con dehiscencia circunsísil, esta especie se ubica en la sección Genlisea A.St.Hil. (Fromm-Trinta, 1979).

Los ejemplares de herbario de Utricularia simulans y $U$. amethystina, entre los que se encontraron los especímenes de Genlisea filiformis, indican que fueron colectados en una sabana con elementos arbóreos destacando Byrsonima crassifolia, Manilkara zapota, Calophyllum brasiliense entre otros, que alcanzan solamente 2 a 3 metros de altura. La cubierta herbácea está compuesta por elementos de las familias Gramineae, Cyperaceae y Rubiaceae principalmente. El suelo es arenoso-pedregoso en el que predomina la grava, de color amarillo rojizo, y la altitud de $220 \mathrm{~m}$. Florece y fructifica de octubre a enero.

Material revisado: México, Chiapas: municipio de Ocosingo, a $16 \mathrm{~km}$ al NW de Boca Lacantum camino a Palenque, sabana, 31-X-1985, E. Martínez 14471B y G. Aguilar (MEXU); misma localidad, 17-I-1986, E. Martínez 16364A y G. Aguilar (MEXU).

Genlisea filiformis ha sido registrada previamente de Belice, Cuba, Nicaragua, Colombia, Venezuela, Guyana, Bolivia y Brasil. En Centroamérica es conocida por varias colectas de Belice (Schipp 607 BM! citado en Gibson, 1974 y Balick et al., 2000; Hunt 204 BM!), de Honduras (Proctor 38796 BM!, Chorley 473 BM!) y de Nicaragua (Stevens 18587 MO! citado en Taylor, 2001); su descubrimiento en México amplía su distribución conocida hasta ahora, y es probable que se encuentre también en el resto de la región centroamericana.

Por lo que respecta a la localidad donde se encontró esta especie en México, cabe hacer notar que se trata de un claro de sabana de aproximadamente $2 \mathrm{~km}$ de largo por $400 \mathrm{~m}$ de ancho, situado en la cresta de una loma, rodeado por selva baja subcaducifolia donde abundan Metopium brownei y Guettarda combsii. En general, en tales rodales se ha colectado poco debido a su apariencia pobre que semeja pastizales degradados, por lo que el conocimiento de su flora es deficiente. Este tipo de vegetación posee especies exclusivas, principalmente de las familias Lentibulariaceae, Burmanniaceae, Droseraceae, Lindsaeaceae, Ophioglossaceae y Gentianaceae, que han sido incorporadas en el estudio de la Flora Mesoamericana.

Cuadro 1. Características distintivas de los géneros de Lentibulariaceae

\begin{tabular}{|l|l|l|l|}
\hline Caracteres & \multicolumn{1}{|c|}{ Pinguicula } & \multicolumn{1}{c|}{ Genlisea } & \multicolumn{1}{c|}{ Utricularia } \\
\hline Estructuras insectívoras & $\begin{array}{l}\text { glándulas sobre las } \\
\text { hojas }\end{array}$ & hojas tubulares & utrículos \\
\hline Flores & flor solitaria & $\begin{array}{l}\text { inflorescencia race- } \\
\text { mosa, ocasionalmen } \\
\text { te flores solitarias }\end{array}$ & $\begin{array}{l}\text { inflorescencia race- } \\
\text { mosa, ocasionalmen } \\
\text { te flores solitarias }\end{array}$ \\
\hline Pedúnculo & ebracteado & bracteado & bracteado \\
\hline Cáliz & 2-partido & 5-partido & 2-partido \\
\hline
\end{tabular}




\section{AGRADECIMIENTOS}

Los autores agradecen al M. en C. Mario Sousa Sánchez y al Dr. Antonio Lot sus valiosos comentarios y sugerencias que mejoraron el trabajo final. Dan las gracias asimismo al Dr. Sergio Zamudio, quien amablemente nos proporcionó información relevante de la distribución de la especie en Centroamérica, y a María del Rosario García Peña por su ayuda en la solicitud de los ejemplares en préstamo.

\section{LITERATURA CITADA}

Balick, M. J., M. H. Nee y D. E. Atha. 2000. Checklist of the vascular plants of Belize. Memoirs of the New York Botanical Garden. Vol. 85. The New York Botanical Garden Press. Nueva York. 246 pp.

Fromm-Trinta, E. 1979. Revisão das espécies do gênero Genlisea St.-Hil. (Lentibulariaceae) das regiõnes Sudeste e Sul do Brasil. Rodriguésia 49: 17-139.

Gibson, D. N. 1974. Lentibulariaceae. In: Standley, P. C. , L. O. Williams y D. N. Gibson. Flora of Guatemala. Fieldiana Bot. 24, Part X: 315-328.

Taylor, P. 2001. Lentibulariaceae. In: Stevens, W. D., C. Ulloa, A. Pool y O. M. Montiel (eds.). Flora de Nicaragua. No. 85, Tomo II. Missouri Botanical Garden, St. Louis Missouri. pp. 1214-1218. 